\title{
The Efficacy of Laser Fluorescence to Detect in Vitro Demineralization and Remineralization of Smooth Enamel Surfaces
}

\author{
Michele Baffi Diniz, D.D.S., M.Sc., ${ }^{1}$ Adriana Franco Paes Leme, D.D.S., M.Sc., Ph.D., ${ }^{2}$ \\ Kátia de Sousa Cardoso, ${ }^{3}$ Jonas de Almeida Rodrigues, D.D.S., M.Sc., Ph.D., ${ }^{1}$ \\ and Rita de Cássia Loiola Cordeiro, D.D.S., M.Sc., Ph.D. ${ }^{4}$
}

\begin{abstract}
Objective: The purpose of this study was to evaluate the efficacy of the laser fluorescence (LF) device in detecting in vitro demineralization and remineralization of smooth surface caries-like lesions. Background Data: The early detection of smooth surface caries-like lesions is important to provide proper management of carious lesions, and allows monitoring of them over time. Also, some authors suggest that LF could be useful in monitoring the caries pathological process. Materials and Methods: Seventy-eight blocks of bovine teeth were obtained, and then submitted to artificial caries lesion induction and to a $\mathrm{pH}$-cycling process. Superficial microhardness (SMH) and laser fluorescence analysis were performed at baseline, after induction of caries-like lesions, and after the $\mathrm{pH}$-cycling regimen to promote remineralization. Results: Friedman's and multiple comparison tests were performed for all variables. SMH analysis showed significant differences $(p<0.05)$ between baseline (286.77 \pm 1.49 Vickers hardness number [VHN] units), before (38.48 $\pm 0.85 \mathrm{VHN})$, and after remineralization $(131.93 \pm 2.63 \mathrm{VHN})$. Baseline values for LF were extremely low $(2.71 \pm 0.05)$, and a statistically significant difference was observed only after remineralization $(3.61 \pm 0.08)$, as demonstrated by the increase in LF values. Conclusion: The LF device did not show efficacy for monitoring in vitro demineralization and remineralization of smooth enamel surfaces.
\end{abstract}

\section{Introduction}

G ENERALLy, EARLY CARIES LESIONS IN ENAMEL are observed clinically as white spots caused by the acid pH of dental plaque. This incipient lesion progresses slowly and has the potential to be remineralized, thus avoiding the need for dental intervention. ${ }^{1,2}$ White spots are opaque because their crystals have altered physical and chemical properties, and the spatial configuration of the crystals and the presence of water reflect changes in the enamel surface compared with the appearance of sound enamel..$^{3,4}$

The early detection of smooth surface caries lesions is important to provide proper management of caries, and allows their monitoring over time. It is well known that conventional methods, such as visual inspection and radiographic examination, are adequate to detect advanced caries le- sions. ${ }^{5-7}$ Quantitative methods have been developed that offer accurate detection, quantification, and monitoring of changes in the mineral content of caries lesions. ${ }^{8}$

The laser fluorescence device (LF; DIAGNOdent, KaVo, Biberach, Germany) is a laser-based instrument that emits red light at a wavelength of $655 \mathrm{~nm}$ from a fiber optic bundle and captures the fluorescence emitted by oral bacterial metabolites. The device is based on the principle that carious tissue fluoresces more strongly than sound tissue, and this change is registered as an increased number displayed on a monitor. ${ }^{9}$

The performance and reproducibility of results of the laser fluorescence device have been investigated for occlusal caries $^{9-13}$ and smooth surface lesions. ${ }^{1,2,8,14,15}$ However, the efficacy of laser fluorescence in monitoring remineralization of incipient caries lesions is still unclear. ${ }^{14,16-18}$

\footnotetext{
${ }^{1}$ Department of Pediatric Dentistry, School of Dentistry of Araraquara, São Paulo State University (UNESP), Araraquara, SP, ${ }^{2}$ Department of Physiological Sciences, School of Dentistry of Piracicaba, State University of Campinas (UNICAMP), Piracicaba, ${ }^{3}$ Department of Pediatric Dentistry, and ${ }^{4}$ Department of Pediatric Dentistry, School of Dentistry of Araraquara, São Paulo State University (UNESP), Araraquara, SP, Brazil.
} 
Thus, the aim of the present study was to evaluate the efficacy of the laser fluorescence device in detecting in vitro deand remineralization of smooth surface caries-like lesions, using a surface microhardness technique which is a recognized method for analyzing de- and remineralization. ${ }^{19}$

\section{Materials and Methods}

\section{Preparation of the enamel blocks}

Two hundred enamel blocks $(4 \times 4 \mathrm{~mm})$ were obtained from bovine incisors and were stored in $2 \%$ formaldehyde solution ( $\mathrm{pH} 7.0$ ) at room temperature. ${ }^{20}$ Each block was embedded in epoxy resin in order to expose only the buccal surface. This procedure was performed to allow polishing of the enamel surface as needed for an appropriate surface microhardness analysis, since such evaluation requires a stable specimen during the indentation process.

The blocks were then stored individually at $100 \%$ humidity. Afterward, the enamel surface of the blocks was serially polished and the surface microhardness analysis was performed using a microhardness tester (Micromet 2100; Buehler Ltd., Lake Bluff, IL, USA) with a Vickers diamond under a $50 \mathrm{-g}$ load for $5 \mathrm{sec} \cdot{ }^{19}$ Five indentations spaced 100 $\mu \mathrm{m}$ from each other were made and the average was recorded. From 200 enamel blocks, only those with hardness of $286.77 \pm 1.49$ Vickers hardness number (VHN) units were selected $(n=152)$ for artificial caries lesion induction. The other blocks (48 enamel blocks) were excluded because their microhardness values had high levels of variation.

\section{Laser fluorescence measurements}

A laser fluorescence device (LF; DIAGNOdent, KaVo, Biberach, Germany) was used according to the manufacturer's instructions. Two operators received training in order to ensure proper assessment of the teeth with the LF device. The operators performed the LF readings using probe tip B, for smooth surfaces. The device was calibrated against a porcelain standard prior to the examination, and then it was recalibrated after testing 10 blocks. ${ }^{16}$ After calibration, each enamel block was taken out of the area with $100 \%$ humidity and gently wiped with a tissue. For each enamel block, the maximum fluorescence value as detected by the device was recorded.

The laser fluorescence readings of the enamel blocks were assessed at baseline, after artificial caries lesion induction, and after the $\mathrm{pH}$-cycling regimen. Each block was analyzed three times by each operator at 1-wk intervals, and the mean values were calculated.

\section{Artificial caries lesion induction}

Caries-like lesions were produced by suspending each enamel block in $32 \mathrm{~mL}$ of a solution containing $0.05 \mathrm{M}$ acetate buffer $50 \%$ saturated with respect to bovine enamel, at $\mathrm{pH} 5.0$ for $16 \mathrm{~h}$ at $37^{\circ} \mathrm{C} .{ }^{21,22}$ To prepare this solution, bovine enamel powder ${ }^{23}$ (particles 74-105 $\mu \mathrm{m}$ in size) was agitated in $0.05-\mathrm{M}$ sodium acetate buffer, $\mathrm{pH} 5.0$, for $96 \mathrm{~h}$ at $37^{\circ} \mathrm{C}(0.50$ $\mathrm{g} / \mathrm{L})$. This saturated solution was filtered and diluted with an equal volume of the same buffer. According to Moreno and Zahradnik, ${ }^{24}$ an acid buffer at $50 \%$ would induce enamel subsurface demineralization without erosion. After demineralization, the surface microhardness (SMH) was assessed again by five indentations spaced $100 \mu \mathrm{m}$ from each other and from the baseline indent. After mean and standard deviation calculation, 78 enamel blocks with known enamel surface microhardness $(38.48 \pm 0.85 \mathrm{VHN})$ were selected for the $\mathrm{pH}$-cycling regimen. The other blocks (74 blocks) were excluded because they showed higher hardness value variations compared to the mean and the standard deviation of all of the blocks.

\section{Treatment and $\mathrm{pH}$-cycling regimen}

The enamel blocks were submitted to dentifrice treatment during the $\mathrm{pH}$-cycling regimen according to White ${ }^{19}$ and Paes Leme et al. ${ }^{21}$ A fluoride dentifrice containing $1100 \mu \mathrm{g}$ F/g, as NaF (Tandy-Colgate, São Paulo, Brazil) was used.

The $\mathrm{pH}$-cycling regimen consisted of 1-min soaks in 100 $\mathrm{mL} \mathrm{33 \%} \mathrm{(w/v)} \mathrm{dentifrice/water} \mathrm{slurries} \mathrm{four} \mathrm{times} \mathrm{per} \mathrm{day}$ (8:00 A.M., 10:00 A.M., 2:30 P.M., and 4:30 P.M.) to simulate toothbrushing exposure. ${ }^{21}$ After each soak the blocks were washed with deionized water. Between the treatments with dentifrice, the enamel blocks were individually immersed in $4 \mathrm{~mL}$ of artificial saliva at $37^{\circ} \mathrm{C}$. The artificial saliva was changed twice a day (8:00 A.M. and 4:30 P.M.). In order to simulate the daily acid challenges occurring in the oral cavity, the enamel blocks were individually immersed in $12 \mathrm{~mL}$ of demineralization solution (with the same composition as the lesion preparation solution) from 12:00 A.M. to 2:00 P.M. The continuous cycles of demineralization and remineralization were carried out for $12 \mathrm{~d}$. On the sixth day the acid demineralizing solution was changed. ${ }^{21}$

After $\mathrm{pH}$ cycling, the SMH of the enamel blocks was measured again. Five indentations spaced $100 \mu \mathrm{m}$ from each other, from the baseline, and from those made after artificial caries lesion induction, were performed. The percentage surface microhardness recovery (SMHR) was individually calculated for each enamel block according to the method of Paes Leme et al.: ${ }^{21}$ (\% hardness recovery $=$ hardness after $\mathrm{pH}$ cycling - hardness after demineralization $\times 100$ /sound enamel hardness - hardness after demineralization). Then the means and the standard deviations of SMHR were calculated.

\section{Statistical analysis}

Intraclass correlation (ICC) was used to assess inter- and intra-examiner reproducibility. The ICC was considered poor when the values were below 0.40 , fair for values between 0.40 and 0.59 , good for values between 0.60 and 0.75 , and excellent for values above $0.75 .^{25}$

The non-parametric Friedman's and multiple comparison tests were performed for LF readings and for SMH analysis at baseline, after artificial caries lesion induction, and after the $\mathrm{pH}$ cycling regimen. The level of significance was set at $5 \%$. The software MedCalc Statistical for Windows (version 9.3.0.0; MedCalc Software, Mariakerke, Belgium) was used to perform the analyses.

\section{Results}

The ICC showed values for inter-examiner reproducibility of 0.78 (baseline), 0.71 (after demineralization), and 0.68 (after remineralization), indicating good to excellent agreement between examinations. For intra-examiner repro- 

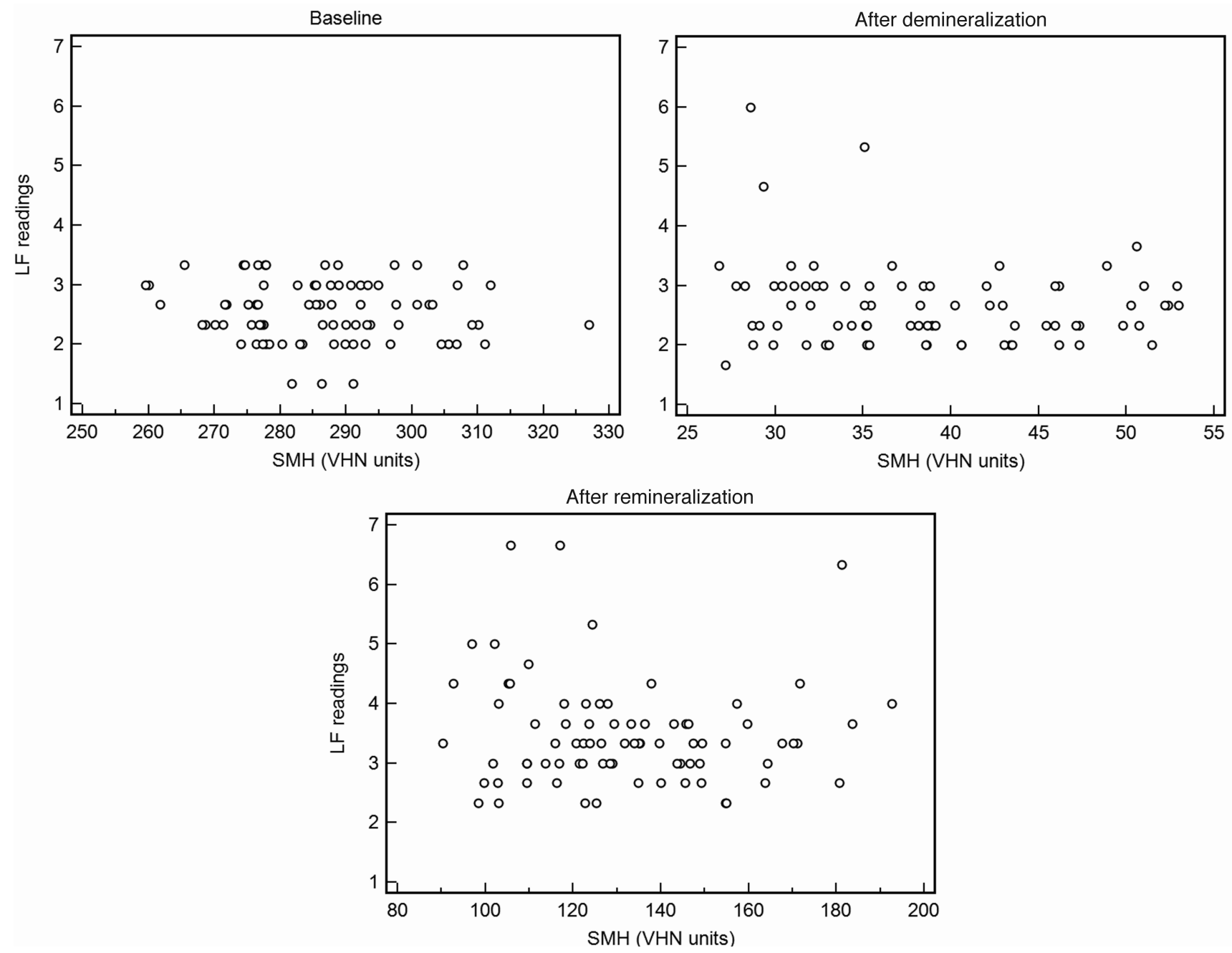

FIG. 1. Scatterplots of the SMH values and LF readings at baseline, after demineralization, and after remineralization.

ducibility, ICC showed reproducibility ranging from 0.51 to 0.61. The scatterplots shown in Fig. 1 represent the dispersion of the LF values according to the microhardness values for the three different periods analyzed.

With regard to the surface microhardness analysis of the enamel blocks, statistically significant differences $(p<0.05)$ were observed among the three periods (Table 1$)$. The percentage of SMHR (means \pm SD) of the enamel blocks treated with fluoride dentifrice was $37.8 \pm 9.9$. The LF readings did not any show significant differences between the values at

Table 1. Enamel Surface Microhardness Analysis AND Laser Fluorescence ReAdings at the Three Study Periods (means \pm SD; $N=78$ )

\begin{tabular}{lrrr}
\hline Method & Baseline & $\begin{array}{c}\text { After } \\
\text { demineralization }\end{array}$ & $\begin{array}{c}\text { After } \\
\text { remineralization }\end{array}$ \\
\hline SMH & $286.77 \pm 1.49^{\mathrm{a}}$ & $38.48 \pm 0.85^{\mathrm{b}}$ & $131.93 \pm 2.63^{\mathrm{c}}$ \\
FL & $2.71 \pm 0.05^{\mathrm{a}}$ & $2.77 \pm 0.6^{\mathrm{a}}$ & $3.61 \pm 0.08^{\mathrm{b}}$ \\
\hline
\end{tabular}

Different letters in the same row indicate significant differences by the multiple comparison test $(p<0.05)$. baseline and after demineralization; however, they had statistically significant differences after remineralization.

\section{Discussion}

Little information about the assessment of remineralization using the LF device is available. Some authors have suggested that the LF device could be useful for monitoring the remineralization process; ${ }^{26,27}$ however, other studies have reported a lack of efficacy of LF in monitoring the remineralization of incipient caries lesions. ${ }^{16,18}$

According to Lussi and Hellwig ${ }^{11}$ a high level of reproducibility of the LF device means that it could be useful for monitoring the carious process. In this study ICC values showed good-to-excellent agreement for the reliability of the LF device in terms of inter-examiner agreement. These results confirmed the high reproducibility reported in previous studies. ${ }^{1,28}$ Nevertheless, intra-examiner agreement showed lower ICC values (from 0.51-0.61). Differing from this, Kühnisch et al., ${ }^{29}$ in an in vitro study assessing occlusal caries, and Aljehani et al., ${ }^{15}$ in an in vivo study assessing orthodontically-induced white spot lesions, showed excellent intra-examiner reproducibility, with ICC values ranging from $0.93-0.98$, and from $0.91-0.99$, respectively. Thus these 
differences may be related to subjective errors in the measurements, such as improper calibration of the device.

The optical effects of mineral tissues, such as reflection, transmission, and color absorption, are subject to change with demineralization of the enamel's microstructure. It has been shown that this mineral loss is related to reflective brightness of the minerals, and this holds considerable promise in the field of quantitative cariology. ${ }^{30}$ According to Ko et al., ${ }^{31}$ variations within the enamel tissue may also affect the scattering coefficient, such as changes in density, crystal size, and prism orientation, from one tooth to another. Tooth reflections show the combined optical effects of the lesion layer and the surrounding sound tissues, which defeats the use of optics for characterization of mineral loss.

Despite the capability of detecting in vivo caries lesion activity, ${ }^{2,15}$ the laser fluorescence device has yielded conflicting results among the various studies. Furthermore, it is important to emphasize that some differences can be found between natural and artificial caries lesions, ${ }^{32}$ although it has been reported that the LF device is able to detect artificial caries lesions induced without bacteria. ${ }^{14}$ The artificial demineralization procedure used in this study is adequate for the study of early enamel caries-like lesions. ${ }^{21,22}$ In the current study, the values obtained with the laser fluorescence device after demineralization were not significantly different from baseline values. This may be related to inadequate detection of small changes in mineral content by the LF device. ${ }^{8,16}$ Mendes and Nicolau ${ }^{14}$ observed an increase in LF values after artificial demineralization, suggesting that it could be due to an increase in the enamel's porosity. Since in our study we used the $\mathrm{SMH}$ as the standard, it is possible that the demineralization process was also observed using this technique; however, it was not detected by the LF device.

On the other hand, an increase in the laser fluorescence readings was observed after the $\mathrm{pH}$-cycling regimen, a finding in agreement with that of Gokalp and Baseren, ${ }^{33}$ who showed that the LF device has good ability to detect sound teeth for in vivo assessments, but an increase in the LF readings was shown immediately after fluoride and chlorhexidine varnish application. However, Mendes et al. ${ }^{16}$ demonstrated that LF readings did not change significantly after in vitro remineralization of natural white spot lesions in primary teeth. Furthermore, in an in vivo study, Silva et al. ${ }^{18}$ observed that LF values did not show any modification after the remineralization of non-cavitated lesions. These results contradict studies that suggest that laser fluorescence could be a useful method for detecting remineralization. ${ }^{15,26,34,35}$

In our study, although a statistically significant increase in LF values was seen after remineralization ( 0.84 units; Table 1 ), it appears that this did not have clinical significance in smooth surfaces, since the LF device can show values varying from 0-99, and values from 0-4 represent arrested carious lesions, and values from 5-99 indicate active lesions. ${ }^{2}$

Another important aspect related to the LF analysis is that some companies are formulating prophylaxis pastes and toothpastes with fluorescent components to provide more aesthetic results for teeth. ${ }^{36}$ The authors discussed the fact that cleaning teeth with such products could leave remnants of the paste in small, narrow areas such as fissures, and it could lead to higher inherent LF values. ${ }^{36}$ In their study assessing occlusal surfaces, only the prophylaxis paste Clinic
(3M, Bioggio, Switzerland) had a significant influence on LF values of sound enamel and carious enamel, but it had no clinical significance. The prophylaxis paste Clinic had a fluorescence value of 4 . However, in our study, the toothpaste used in the $\mathrm{pH}$-cycling regimen (Tandy-Colgate, São Paulo, Brazil) had a fluorescence value of 1 . For this reason, the increase in LF values observed after remineralization was not related to fluorescence present in the toothpaste.

Regarding the SMH analysis, it was observed that $37 \%$ of the enamel's surface recovered after $\mathrm{pH}$ cycling in the presence of artificial saliva and a fluoridated dentifrice, as was previously shown by White, ${ }^{19}$ Maia et al., ${ }^{37}$ and Paes Leme et al. ${ }^{21}$ White ${ }^{19}$ and Paes Leme et al. ${ }^{21}$ suggested that this technique would be an excellent means of simple and accurate assessment of remineralization in incipient caries lesions. In addition, as it is a non-destructive technique and does not damage the enamel's structure, ${ }^{19}$ the microhardness test was the method chosen for this longitudinal study.

Thus, in our study it we found a mean of $37 \%$ of SMHR, and it cannot be dismissed that the increase in LF readings after remineralization may be related to a decrease in light scattering due to remineralization and the subsequent decrease in porosity. We also observed that all LF values obtained in our study in the three study periods remained within the same range (from 2-4) for all the different carious states. It has been suggested that changing LF readings reflect changes in the organic content (proto- and meso-porphyrins) rather than the inorganic content of the tooth substance. 1,8,9,38 These porphyrins are produced by bacteria inside carious tooth substance, and may be released from bacteria outside the tooth and diffuse into the tooth. Therefore it may be argued that LF does not measure remineralization directly. More effective toothbrushing would mean less plaque, and thus less bacteria to produce porphyrins externally, and as a consequence, reduces laser fluorescence. In addition, fluoride treatment may result in a reduction of porosity of the tooth surface, and consequently less porphyrin diffuses into the enamel. ${ }^{35}$

\section{Conclusion}

In this study, demineralization and remineralization were detected by surface microhardness analysis. However, laser fluorescence did not prove efficacious in detecting in vitro demineralization and remineralization of smooth enamel surfaces. Further in vitro studies using bacterial caries models, as well as in vivo studies are needed to elucidate whether this device is able to detect natural or induced caries-like lesions and their remineralization.

\section{Disclosure Statement}

No conflicting financial interests exist.

\section{References}

1. Shi, X.Q., Tranaeus, S., and Angmar-Mansson, B. (2001). Comparison of QLF and DIAGNOdent for quantification of smooth surface caries. Caries. Res. 35, 21-26.

2. Pinelli, C., Campos Serra, M., and de Castro Monteiro Loffredo, L. (2002). Validity and reproducibility of a laser fluorescence system for detecting the activity of white-spot lesions on free smooth surfaces in vivo. Caries Res. 36, 19-24. 
3. Angmar-Mansson, B., and ten Bosch, J.J. (1987). Optical methods for the detection and quantification of caries. Adv. Dent. Res. 1, 14-20.

4. Barbakow, F., Imfeld, T., and Lutz, F. (1991). Enamel remineralization: how to explain it to patients. Quintessence Int. $22,341-347$.

5. Kidd, E.A. (1984). The diagnosis and management of the early carious lesions in permanent teeth. Dent. Update 11, 69-78.

6. Angmar-Mansson, B. (2001). How to measure the effects of fluoride treatments in clinical trials? Assessment: modern versus traditional methods. Caries Res. 35, 30-33.

7. Nyvad, B. (2004). Diagnosis versus detection of caries. Caries Res. 38, 192-198.

8. Shi, X.Q., Tranaeus, S., and Angmar-Mansson, B. (2001). Validation of DIAGNOdent for quantification of smooth surface caries: an in vitro study. Acta Odontol. Scand. 59, 74-78.

9. Lussi, A., Inwinkelried, S., Pitts, N., Longbottom, C., and Reich, E. (1999). Performance and reproducibility of a laser fluorescence system for detection of occlusal caries in vitro. Caries Res. 33, 261-266.

10. Lizarelli, R.F., Bregagnolo, J.C., Lizarelli, R.Z., Palhares, J.M., and Villa, G.E. (2004). A comparative in vitro study to diagnose decayed dental tissue using different methods. Photomed. Laser Surg. 22, 205-210.

11. Lussi, A., and Hellwig, E. (2006). Performance of a new laser fluorescence device for the detection of occlusal caries in vitro. J. Dent. 34, 467-471.

12. Rodrigues, J.A., Diniz, M.B., Josgrilberg, E., and Cordeiro, R.C.L. (2008). In vitro comparison of laser fluorescence performance with visual examination for detection of occlusal caries in permanent and primary molars. Lasers Med. Sci. [Epub ahead of print].

13. Diniz, M.B., Rodrigues, J.A., Paula, A.B., and Cordeiro, R.C.L. (2008). In vivo evaluation of laser fluorescence performance using different cut-off limits for occlusal caries detection. Lasers Med. Sci. [Epub ahead of print].

14. Mendes, F.M., and Nicolau, J. (2004). Utilization of laser fluorescence to monitor caries lesions development in primary teeth. J. Dent. Child. 71, 139-142.

15. Aljehani, A., Bamzahim, M., Yousif, M.A., and Shi, X.Q. (2006). In vivo reliability of an infrared fluorescence method for quantification of carious lesions in orthodontic patients. Oral Health Prev. Dent. 4, 145-150.

16. Mendes, F.M., Nicolau, J., and Duarte, D.A. (2003). Evaluation of the effectiveness of laser fluorescence in monitoring in vitro remineralization of incipient caries lesions in primary teeth. Caries Res. 37, 442-444.

17. Mendes, F.M., Siqueira, W.L., Mazzitelli. J.F., Pinheiro, S.L., and Bengtson, A.L. (2005). Performance of DIAGNOdent for detection and quantification of smooth-surface caries in primary teeth. J. Dent. 33, 79-84.

18. Silva, B.B., Severo, N.B., and Maltz, M. (2007). Validity of diode laser to monitor carious lesions in pits and fissures. J. Dent. 35, 679-682.

19. White, D.J. (1987). Reactivity of fluoride dentifrices with artificial caries I. Effect on early lesions: F uptake, surface hardening and remineralization. Caries Res. 21, 126-140.

20. Delbem, A.C., and Cury, J.A. (2002). Effect of application time of APF and NaF gels on microhardness and fluoride uptake of in vitro enamel caries. Am. J. Dent. 15, 169-172.

21. Paes Leme, A.F., Tabchoury, C.P., Zero, D.T., and Cury, J.A. (2003). Effect of fluoridated and acidulated phosphate fluoride application on early artificial carious lesions. Am. J. Dent. 16, 91-95.
22. Vieira, A.E., Delbem, A.C., Sassaki, K.T., Rodrigues, E., Cury, J.A., and Cunha, R.F. (2005). Fluoride dose response in $\mathrm{pH}$-cycling models using bovine enamel. Caries Res. 39, 514-520.

23. Asgar, K. (1956). Chemical analysis of human teeth. J. Dent. Res. 35, 742-748.

24. Moreno, E.C., and Zahradnik, R.T. (1974). Chemistry of enamel subsurface demineralization in vitro. J. Dent. Res. 53, 226-235.

25. Lin, I.K. (1989). A concordance correlation coefficient to evaluate reproducibility. Biometrics 45, 255-268.

26. Ross, G. (1999). Caries diagnosis with the DIAGNOdent laser: A user's product evaluation. Ont. Dent. 76, 21-24.

27. Ferreira, J.M.S., Mendes, A.C.R., Silva, M.F.A., Oliveira, A.F.B., and Sampaio, F.C. (2006). Use of laser fluorescence for monitoring white spot lesions in smooth surfaces under fluoride therapy. Caries Res. 40, 344.

28. Tranaeus, S., Lindgren, L.E., Karlsson, L., and AngmarMansson, B. (2004). In vivo validity and reliability of IR fluorescence measurements for caries detection and quantification. Swed. Dent. J. 28, 173-182.

29. Kühnisch, J., Ziehe, A., Brandstädt, A., and HeirinchWeltzien, R. (2004). An in vitro study of the reliability of DIAGNOdent measurements. J. Oral Rehabil. 31, 895899.

30. ten Bosch, J.J., van der Mai, H.C., and Borsboom, P.C. (1984). Optical monitor of in vitro caries. A comparison with chemical and microradiographic determination of mineral loss in early lesions. Caries Res. 18, 540-547.

31. Ko, C.C., Tantbirojn, D., Wang, T., and Douglas, W.H. (2000). Optical scattering power for characterization of mineral loss. J. Dent. Res. 79, 1584-1589.

32. Marsh, P.D. (1995). The role of microbiology in models of dental caries. Adv. Dent. Res. 9, 244-254.

33. Gokalp, S., and Baseren, M. (2005). Use of laser fluorescence in monitoring the durability and cariostatic effects of fluoride and chlorhexidine varnishes on occlusal caries: A clinical study. Quintessence Int. 36, 183-189.

34. Andersson, A., Skold-Larsson, K., Petersson, L., and Twetman, S. (2004). Measurement of enamel lesion regression with a laser fluorescence device (DIAGNOdent): A pilot study. Orthodontics 1, 201-205.

35. Shirrmeister, J.F., Gebrande, J.P., Altenburger, M.J., Möntig, J.S., and Hellwig, E. (2007). Effect of dentifrice containing 5000 ppm fluoride on non-cavitated fissure carious lesions in vivo after 2 weeks. Am. J. Dent. 20, 212-216.

36. Lussi, A., and Reich, E. (2005). The influence of toothpastes and prophylaxis pastes on fluorescence measurements for caries detection in vitro. Eur. J. Oral Sci. 113, 141-144.

37. Maia, L.C., de Souza, I.P., and Cury, J.A. (2003). Effect of a combination of fluoride dentifrice and varnish enamel surface rehardening and fluoride uptake in vitro. Eur. J. Oral Sci. 111, 68-72.

38. Lussi, A., Hibst, R., and Paulus, R. (2004). DIAGNOdent: An optical method for caries detection. J. Dent. Res. 83, C80-C83.

Address reprint requests to: Dr. Michele Baffi Diniz, D.D.S., M.Sc. Faculdade de Odontologia de Araraquara-Departamento de

Clínica Infantil

Rua Humaitá, 1680, CEP 14801-903

Araraquara, SP, Brazil

E-mail: mibdiniz@hotmail.com 